\title{
Erratum to: Entropy of self-gravitating anisotropic matter
}

\author{
Hyeong-Chan Kim ${ }^{\mathrm{a}}$, Youngone Lee $^{\mathrm{b}}{ }_{\mathbb{C}}$
}

School of Liberal Arts and Sciences, Korea National University of Transportation, Chungju 380-702, Korea

Received: 29 October 2019 / Accepted: 8 November 2019 / Published online: 27 November 2019

(C) The Author(s) 2019

Erratum to: Eur. Phys. J. C (2019) 79:679

https://doi.org/10.1140/epjc/s10052-019-7189-2

1. The Eq. (45) should read

$$
s=s_{0} \rho^{1-\frac{1}{2 w_{2}}} r^{-\frac{1+w_{2}}{w_{2}}} \sqrt{\frac{1-2 m / r}{1-2 \bar{m} / r}},
$$

where $\bar{m}=m-M$.

2. Contrary to the Lagrangian (37) which is deduced from the first principle, there is no guarantee that the trial Lagrangian (39) represents the true entropy for $w_{1}=-1$ although it matches well for $w_{1} \neq-1$ cases.

The Lagrangian corresponding to $a, b$ in Eq. (44) is

$\mathcal{L} \propto\left(\bar{m}^{\prime}\right)^{1-\frac{1}{2 w_{2}}} r^{-1} / \sqrt{1-2 \bar{m} / r}$.

However, if the solutions are analytic around $w_{1}=-1$, the $w_{1} \rightarrow-1$ limit of the Lagrangian (37) gives

$$
\lim _{w_{1} \rightarrow-1} \mathcal{L} \propto\left(m^{\prime}\right)^{1-\frac{1}{2 w_{2}}} r^{-1}
$$

which is different from the above Lagrangian. Thus, one should be cautious in using the entropy density for $w_{1}=-1$.

Therefore, the exact form of the entropy density for $w_{1}=-1$ should be derived by taking $w_{1} \rightarrow-1$ limit of the entropy function Eq. (47). The entropy density so obtained has the form

$s=s_{0} \rho^{1-\frac{1}{2 w_{2}}} r^{-\frac{1+w_{2}}{w_{2}}} \sqrt{1-2 m / r}$.

Open Access This article is distributed under the terms of the Creative Commons Attribution 4.0 International License (http://creativecomm ons.org/licenses/by/4.0/), which permits unrestricted use, distribution, and reproduction in any medium, provided you give appropriate credit to the original author(s) and the source, provide a link to the Creative Commons license, and indicate if changes were made. Funded by SCOAP ${ }^{3}$.

The original article can be found online at https://doi.org/10.1140/ epjc/s10052-019-7189-2.

a e-mail: hyeongchan@gmail.com

be-mail: youngone@ut.ac.kr 